\title{
Epithelial fibroblast triggering and interactions in pulmonary fibrosis
}

\author{
P.W. Noble
}

ABSTRACT: Idiopathic pulmonary fibrosis (IPF) is characterised by repeated injury to the alveolar epithelium with loss of lung epithelial cells and abnormal tissue repair, resulting in excessive accumulation of fibroblasts and myofibroblasts, deposition of extracellular matrix components and distortion of lung architecture, eventually leading to respiratory failure.

There is growing circumstantial evidence to suggest that in IPF the alveolar epithelium is prone to undergoing programmed cell death following repeated injury, although the mechanism for inducing epithelial apoptosis is, as yet, unknown. Potentially, one explanation may be the formation of misfolded proteins and an unfolded protein response-mediated apoptosis in alveolar epithelial cells (AECs), in response to abnormal protein production and aggregation.

Epithelial apoptosis is accompanied by damage to the basement membrane leading to the release of growth factors and chemokines, which recruit fibroblasts to the site of injury (fibroblastic foci). Instead of AECs healing by repair, myofibroblast proliferation and extracellular matrix deposition continues unabated in IPF.

The transformation of epithelial cells into mesenchymal cells, a process known as epithelialmesenchymal transition, which allows direct communication between cells, is a possible explanation for the activation of alveolar epithelial cells in idiopathic pulmonary fibrosis. The present article discusses this process and other potential mechanisms by which epithelial cell injury can lead to fibroblast recruitment and accumulation in idiopathic pulmonary fibrosis.

KEYWORDS: Alveolar epithelial cells, apoptosis, epithelial-mesenchymal transition, misfolded proteins, unfolded protein response

diopathic pulmonary fibrosis (IPF), a rare, progressive interstitial lung disease (ILD) is the most common and most lethal of the idiopathic interstitial pneumonias [1]. It is characterised histologically by a pattern of usual interstitial pneumonia (UIP), in which the peripheral subpleural parenchyma shows evidence of fibroblastic foci and microscopic honeycombing interspersed with areas of normal or nearly normal lung tissue (fig. 1) [1]. There is evidence to suggest that IPF results from repeated cycles of alveolar epithelial cell (AEC) injury and apoptosis in a process akin to abnormal wound healing [2]. The present article will: 1) review the concepts surrounding AEC injury in IPF; 2) discuss potential mechanisms by which epithelial cell injury can lead to fibroblast recruitment and accumulation; and 3) present a potential unifying hypothesis to account for the process of epithelial dysfunction in IPF.

\section{EPITHELIAL INJURY HYPOTHESIS}

The search for an alternative hypothesis to unremitting, chronic inflammation as the primary explanation for the pathophysiology of IPF derives, in part, from the lack of therapeutic efficacy of high-dose immunosuppressive therapy in patients with IPF. This is in contrast to other ILDs, such as sarcoidosis, hypersensitivity pneumonitis, nonspecific interstitial pneumonia and desquamative interstitial pneumonia, where potent corticosteroid therapy has proved to be effective. Moreover, inflammation is not a prominent histopathological feature of UIP.

The inflammatory (alveolitis) hypothesis of IPF, which was dominant in the 1970s and 1980s, has since been challenged by the epithelial injury hypothesis, in which fibrosis is believed to result from epithelial injury, activation and/or apoptosis with abnormal wound healing in the absence of chronic inflammation [3-5]. This hypothesis suggests that recurrent unknown injury to distal pulmonary parenchyma causes repeated epithelial injury and apoptosis. The resultant loss of alveolar epithelium exposes the underlying basement membrane to oxidative damage and degradation (fig. 2) [6]. This process appears to be unique to UIP. For example, in other diseases where lung injury occurs, such as acute lung
CORRESPONDENCE

P.W. Noble

Division of Pulmonary

Allergy and Critical Care Medicine Duke University Medical Center MSRBII

106 Research Drive DUMC Box 103000

Durham

NC 27710

USA

Fax: 19196845389

E-mail: paul.noble@duke.edu

STATEMENT OF INTEREST

P.W. Noble serves, or has served, as a consultant to Intermune Pharmaceuticals, Actelion Pharmaceuticals, Gilead, Novartis Wyeth, Boehringer Ingelheim and Genzyme in the development of clinical trials for idiopathic pulmonary fibrosis. P.W. Noble also received an unrestricted grant in 2003 from Intermune

Pharmaceuticals. 


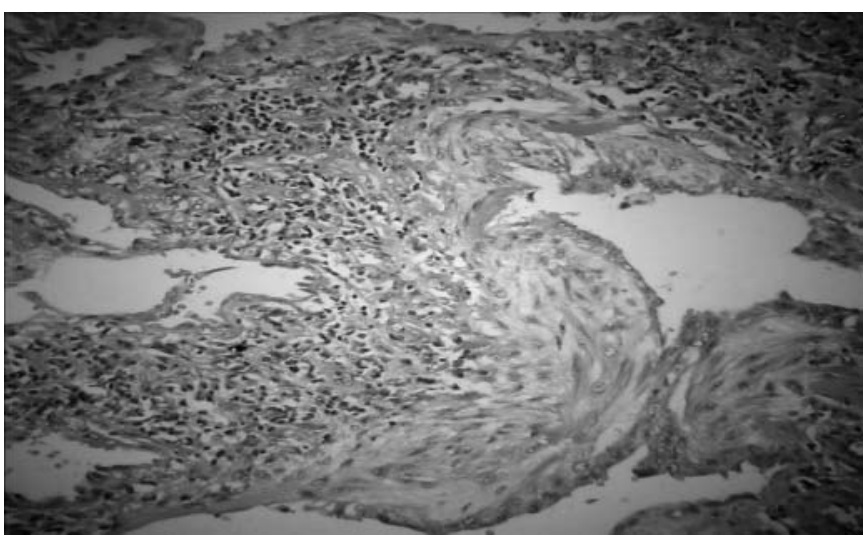

FIGURE 1. Histology slide of a lung section showing evidence of fibroblastic foci, which is a cardinal feature of usual interstitial pneumonia.

injury or diffuse alveolar damage, if the patient survives then the lung essentially recovers normally. This does not appear to be the case in UIP and potentially this failure of reepithelialisation provides a stimulus for persistent pro-fibrotic growth factor production, fibroblast proliferation, excessive extracellular matrix (ECM) deposition and progressive fibrosis.
The resultant distortion of lung architecture also affects the pulmonary vasculature. These changes are understood to be some of the primary changes underlying dyspnoea in IPF.

Evidence of this theory has been established from numerous animal studies, which have shown that exposure to bleomycin causes AEC injury, necrosis and apoptosis [7, 8], whereas inhibiting apoptosis reduces fibrosis and subsequent collagen deposition [9]. In response to bleomycin-induced lung injury, fibrosis is reduced in the absence of pro-apoptotic proteins, such as the Bcl-2 homology (BH3) interacting domain death agonist (BID) and early growth response-1. For example, mice lacking the pro-apoptotic Bcl-2 family member BID display significantly less pulmonary fibrosis in response to bleomycin compared with controls, despite similar levels of inflammation, lung injury and active pro-fibrotic transforming growth factor (TGF)- $\beta 1$ [10]. Under certain circumstances, TGF- $\beta 1$ can make AECs more sensitive to apoptosis and thus promote a pro-apoptotic pathway. There is also immunohistochemical evidence of increased expression of pro-apoptotic proteins in the alveolar epithelium of IPF patients [11].

Clearly, there is growing circumstantial evidence to suggest that in IPF the alveolar epithelium is prone to undergoing programmed cell death, although the mechanism for inducing

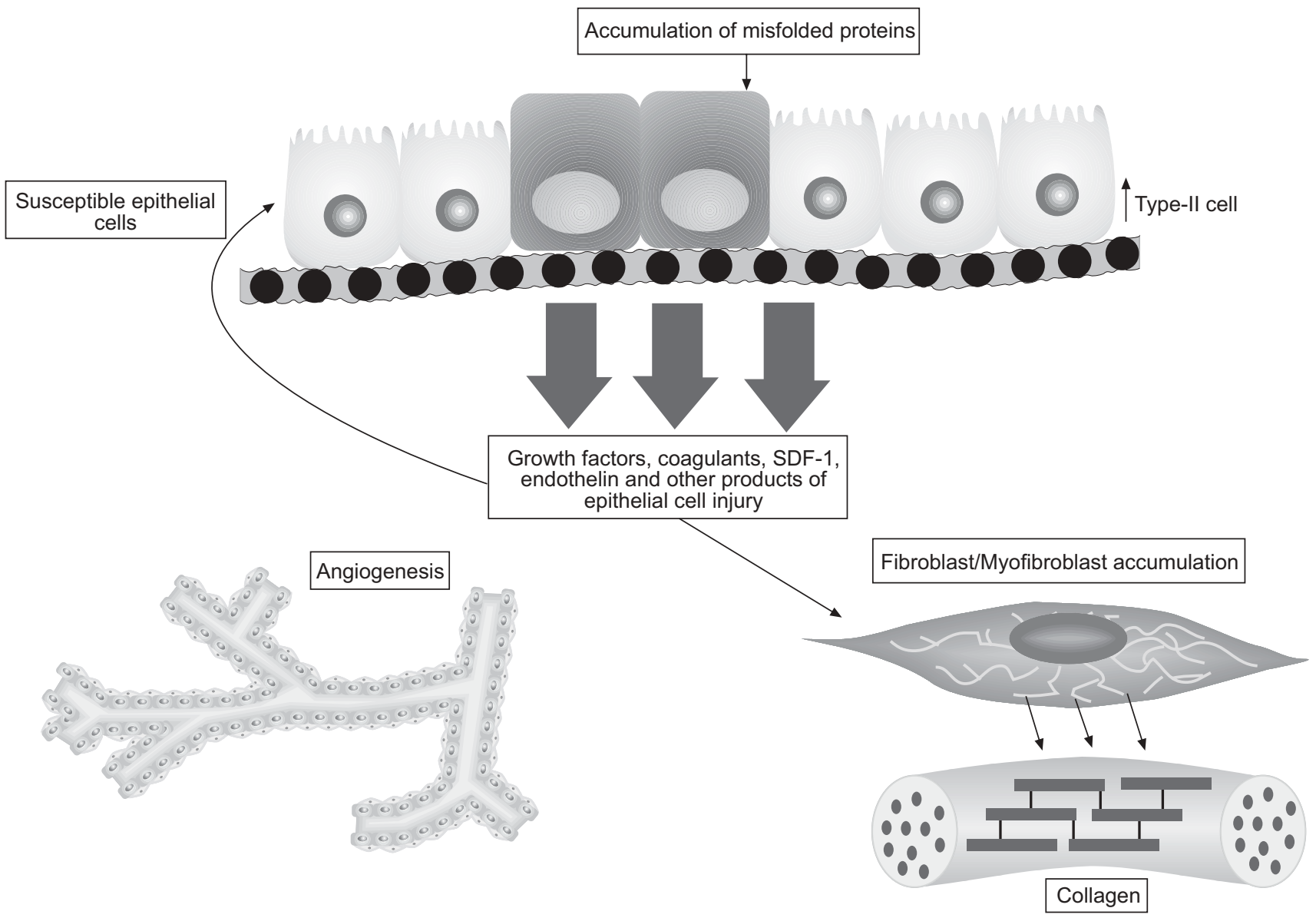

FIGURE 2. Diagrammatic representation of the epithelial injury hypothesis of idiopathic pulmonary fibrosis in which fibrosis results from abnormal wound healing in response to repeated alveolar epithelial cell injury in the relative absence of inflammation. SDF-1: stromal cell-derived factor-1. Modified from [6] with permission from the publisher. 

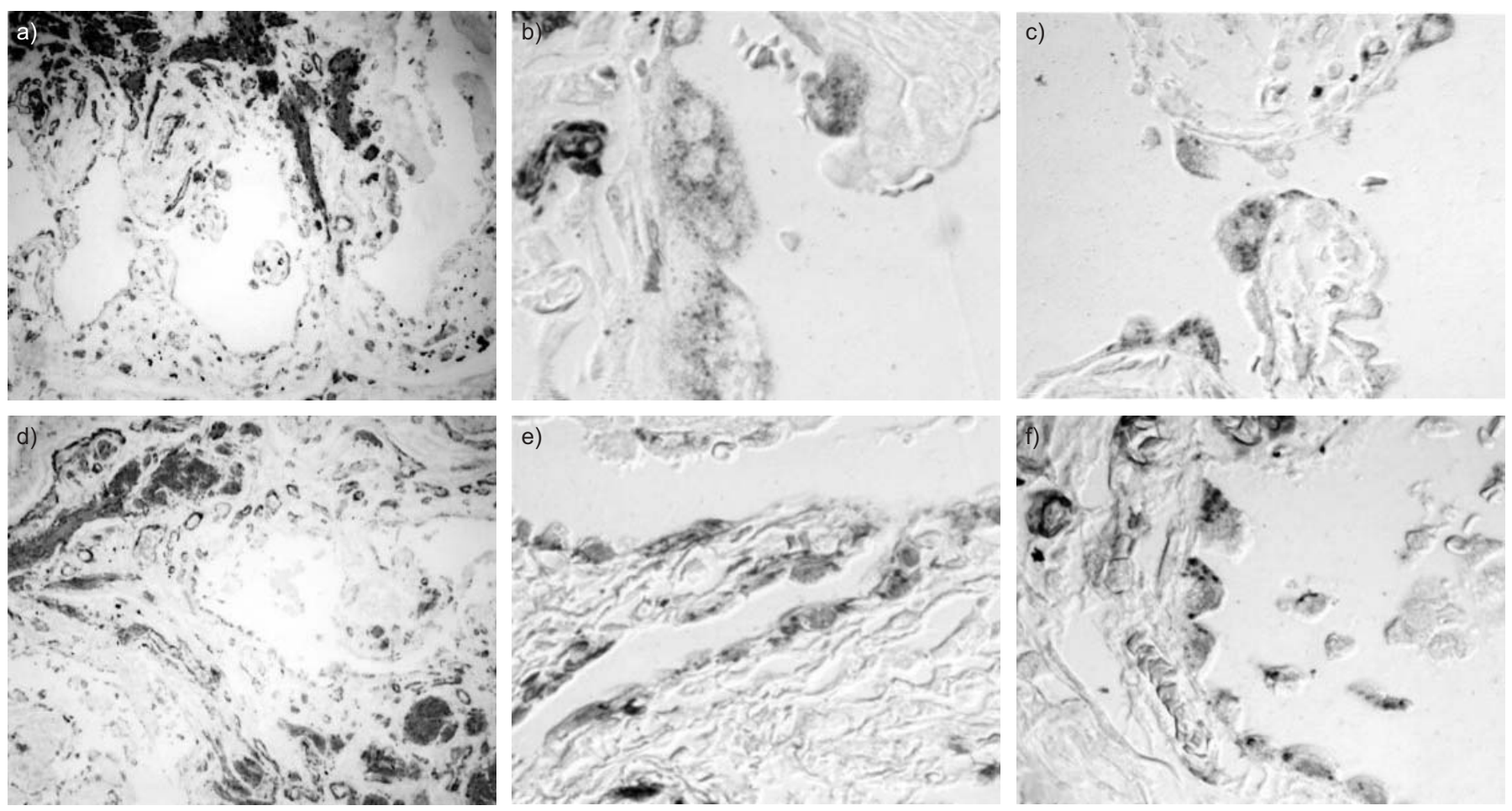

FIGURE 3. Histological evidence of alveolar epithelial cells undergoing epithelial-mesenchymal transition in response to transforming growth factor- $\beta 1$. a-c) $\alpha$-smooth muscle actin ( $\alpha$-SMA) and pro surfactant protein (SP)-B or $\mathrm{d}-\mathrm{f}$ ) $\alpha$-SMA and thyroid transcription factor-1. a, d) Low-power images reveal severe destruction of normal lung architecture due to fibrosis. High-power images (two representative fields from within the low-power field shown) reveal abundant epithelial cells adjacent to $b$, $c$ ) fibroblastic foci co-expressing SP-B and $\alpha$-SMA or e, f) thyroid transcription factor-1 and $\alpha$-SMA. Reproduced from [13] with permission from the publisher.

epithelial apoptosis is, as yet, unknown. Epithelial apoptosis is accompanied by damage to the basement membrane leading to the release of growth factors and chemokines. As discussed below, it appears that some of the products of epithelial cell injury may stimulate fibroblasts to produce ECM components, most notably collagen, that can lead to dysregulated repair of the epithelial/endothelial barrier.

\section{EPITHELIAL CELL ACTIVATION}

The precise process by which AECs become "activated" and communicate with fibroblasts leading to unremitting fibrosis in IPF remains poorly understood. Nonetheless, various theories have been proposed to explain this process, including the production of fibroblast chemotactic factors by activated AECs and the release of growth factors involved in reepithelisation. These growth factors include TGF- $\beta 1$, platelet derived growth factor, insulin-like growth factor-1 and endothelin, among others. AECs may also be a source of activating TGF- $\beta 1$ (aV $\beta 6$ integrin); for example, knockout mice devoid of av $\beta 6$ integrin are unable to activate latent TGF- $\beta 1$ and, as such, are entirely protected from bleomycin-induced pulmonary fibrosis [12]. Thus, when the distal epithelium in the lung becomes injured and the basement membrane loses its integrity, it endeavours to re-epithelialise the surface and, as a result, growth factors are produced that can potentially recruit fibroblasts or myofibroblasts. Thus, instead of healing by repair, myofibroblast proliferation and ECM deposition continues unabated.
The transformation of epithelial cells into mesenchymal cells, which leads to direct communication between cells, is another possible explanation for AEC activation. This so-called epithelial-mesenchymal transition (EMT) is supported by data from several studies $[13,14]$. Chronic exposure of rat primary lung-epithelial cells to TGF- $\beta 1$, which is critical to the progression of fibrosis in IPF, was found to result in increased expression of mesenchymal cell markers, such as $\alpha$-smooth muscle actin ( $\alpha$-SMA), type-I collagen, vimentin and desmin, with concomitant transformation to fibroblast-like morphology (fig. 3) [13]. Immunohistochemical staining of lung tissue from patients with IPF has shown abundant evidence of co-localisation of epithelial markers, such as thyroid transcription factor-1, which is specific for lung epithelium, and $\alpha$-SMA, a critical determinant of myofibroblasts [13]. Therefore, both in vitro and ex vivo evidence exist to suggest that lung epithelial cells can undergo the transition from epithelial to a myofibroblast-like morphology [13]. An experimental study in a mouse model of pulmonary fibrosis probably best exemplifies that EMT can occur in vivo [14]. In the study by KIM et al. [14], the fate of lung epithelial cells genetically altered to permanently express $\beta$ galactosidase was followed after the animals were exposed to TGF- $\beta 1$. Results showed that over a 3-week period a surprisingly large percentage of $\beta$-galactosidase expressing cells had acquired mesenchymal characteristics. It was notable that the increase in vimentin-positive cells within injured lungs was almost exclusively $\beta$-galactosidase positive, indicating that epithelial cells were the main source of mesenchymal cells in 

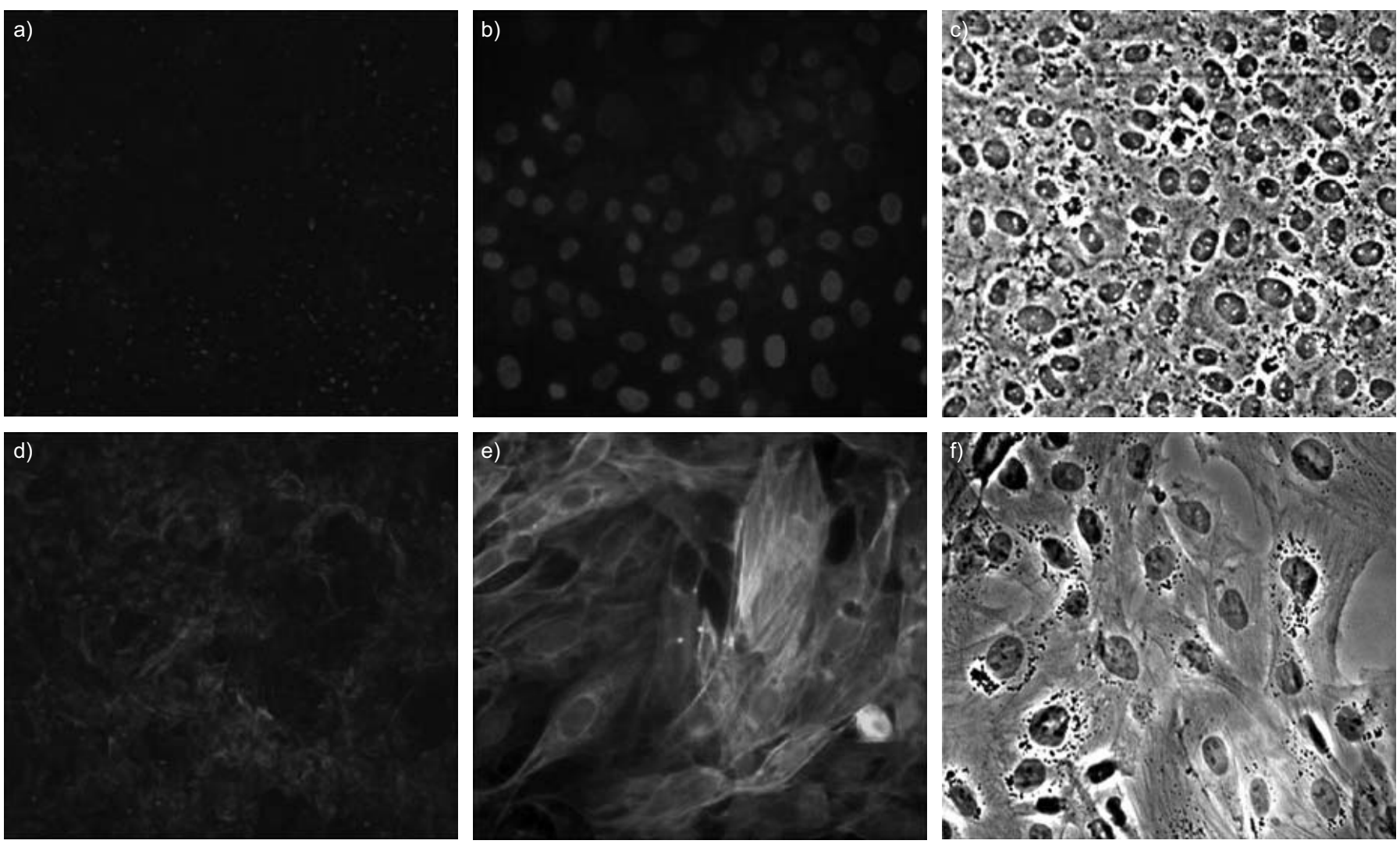

FIGURE 4. Histological evidence for endothelin (ET)-1 induction of epithelial-mesenchymal transition in rat primary distal epithelial cells. a-c) Control, d-f) ET-1. Immunofluorescence analysis demonstrated that ET-1 increases $\alpha$-smooth muscle actin ( $\alpha$-SMA) expression, and that the increase is inhibited by BQ-123, an ET-A antagonist, but not BQ-788 an ET-B antagonist. a, d) $\alpha$-SMA stained with DAPI. b, e) $\alpha$-SMA stained with DAPI showed prominent intracellular stress fibres. c, f) Alveolar epithelial cells treated with ET-1 also changed morphology from a typical rounded shape to an elongated fibroblast-like morphology (phase images). BQ-123 prevented this morphological change, while BQ-788 did not. Reproduced from [15] with permission from the publisher.

the mouse model of pulmonary fibrosis. The fact that this process also required an injured matrix was notable; in effect, matrix that develops in the context of injury was required for EMT such that EMT only occurred at sites of focal injury.

More recently, JAIN et al. [15] demonstrated that endothelin-1 (ET)-1 may play a central role in the induction of EMT. In this in vitro study, rat primary distal-epithelial cells were found to express high levels of ET type $\mathrm{A}$ receptors $\left(\mathrm{ET}_{\mathrm{A}}\right)$ and, to a lesser extent, type $\mathrm{B}$ receptors $\left(\mathrm{ET}_{\mathrm{B}}\right)$, suggesting an autocrine or paracrine function for alveolar ET-1. Primary AECs were found to produce ET-1 at physiologically active levels, via $\mathrm{ET}_{\mathrm{A}}$ activation, leading to increased synthesis of TGF- $\beta 1$ and the induction of EMT in AECs (figs 4 and 5) [15]. Interestingly, ET-1-induced EMT was attenuated by a TGF- $\beta 1$ neutralising antibody [15]. Although, to date, there is no in vivo evidence to show that ET-1 is a critical determinate of EMT, in vitro studies certainly suggest that it might be possible.

\section{ENDOPLASMIC RETICULUM STRESS AND THE UNFOLDED PROTEIN RESPONSE IN IPF}

Stresses within the endoplasmic reticulum (ER), the site of protein synthesis within the cell, can lead to protein misfolding and aggregation. To prevent misfolded protein-aggregation, the ER activates the unfolded protein response (UPR). This signal transduction cascade allows for: 1) an increase in capacity for protein folding; 2) a reduction in newly translated proteins entering the ER; and 3) an increase in the breakdown of misfolded proteins. Ultimately, the UPR signals cell apoptosis. Aberrant protein folding is known to occur in lung diseases such as cystic fibrosis and $\alpha_{1}$-antitrypsin deficiency, where mutations in proteins become dysregulated because of protein misfolding and are then prematurely degraded in the ER [16]. Evidence is emerging to suggest that misfolding and abnormal processing of proteins in the lung may also occur in various ILDs, including IPF [16].

The fibrosis that characterises IPF only occurs in the lung, and the lung is the major source of surfactant proteins (SPs), such as SP-C. This protein appears vulnerable to mutations that disrupt folding and secretion [16]. For example, mutations within the BRICHOS domain of SP-C have been linked to ILD and can cause misfolding of the SP-C proprotein [17]. In addition, a number of genes known to be involved in the UPR are significantly upregulated in patients with UIP, the histological pattern associated with IPF. These include mitogen-activated protein kinase-3-kinase-5, eukaryotic translation initiation factor (Eif) $2-\alpha$ kinase 3 (Eif2AK3), activating transcription factor (ATF)-6, caspase 5 apoptosis-related cystein protease, Eif 2, $2 \beta$ (Eif2s2) and DNA-damage-inducible transcript 3 (i.e. CCAAT/enhancer binding protein homologous protein; $\mathrm{CHOP}$ ), all of which have functional significance in the ER stress response and apoptosis. 

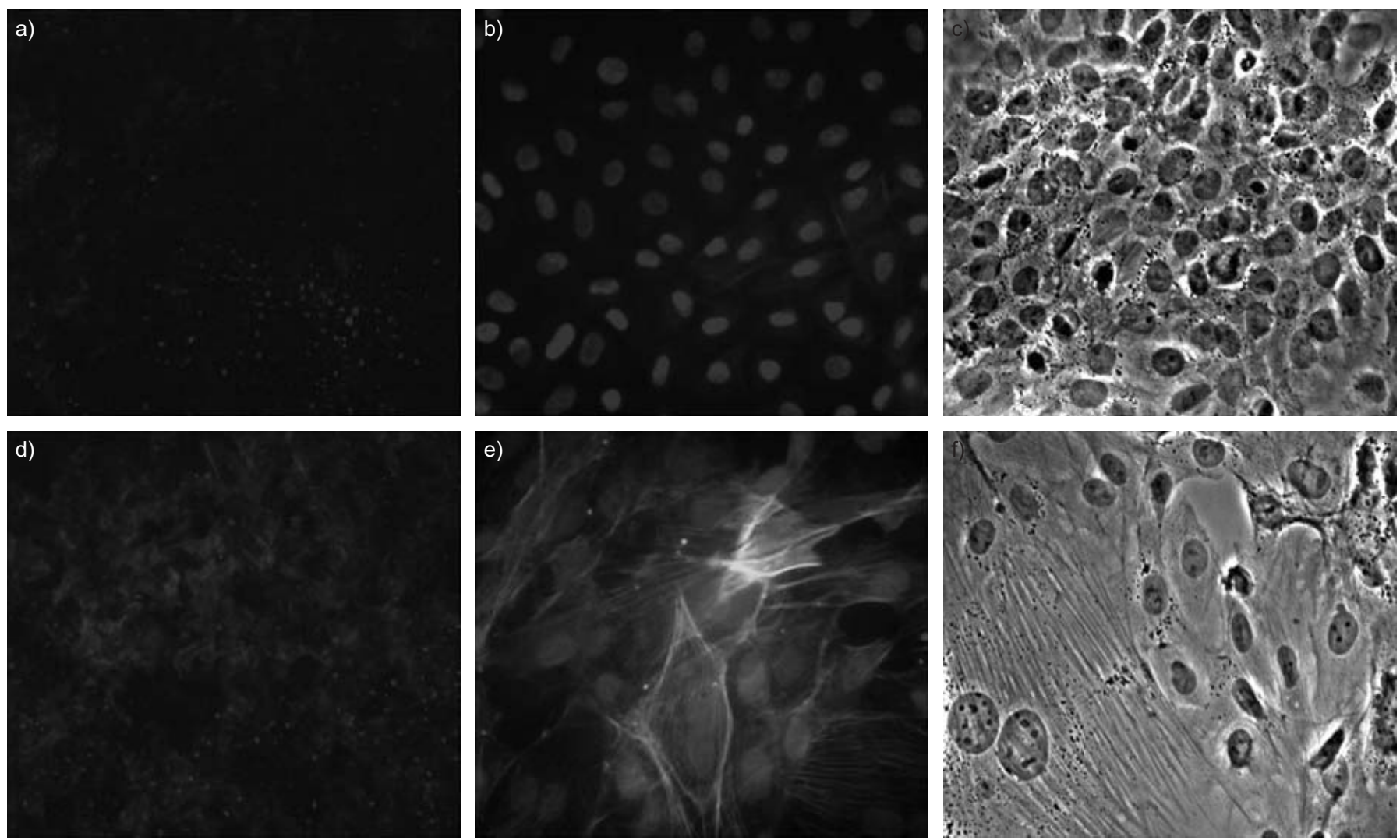

FIGURE 5. Histological evidence for endothelin (ET)-1 induction of epithelial-mesenchymal transition in rat primary distal epithelial cells. a-c) ET-1 plus BQ-123, and d-f) ET-1 plus BQ-788. Immunofluorescence analysis demonstrated that ET-1 increases $\alpha$-smooth muscle actin ( $\alpha$-SMA) expression, and that the increase is inhibited by BQ-123,

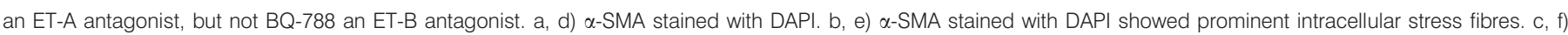

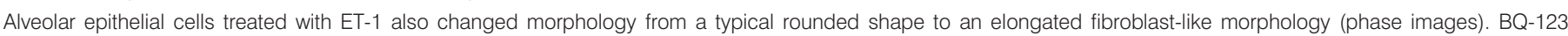
prevented this morphological change, while BQ-788 did not. Reproduced from [15] with permission from the publisher.

Under normal circumstances when misfolded protein begins to accumulate, binding immunoglobulin protein $(\mathrm{BiP})$, or glucose regulated protein 7 as it is also known, is released from ATF-6, inositol requiring kinase- 1 or protein kinase R-related ER kinase. Acting as a chaperone it dissociates from the ER and tries to refold the misfolded proteins. In essence, there is an adaptive response that aims to: 1) deal with ER stress; 2) shut off new protein synthesis; and 3) restore ER homeostasis. Once BiP moves into the ER, cell surface regulators, all of which are identified from microarray analysis, are upregulated to specifically activate a pathway to shut-off translation and synthesise new chaperones (fig. 6a) [18]. If this process works normally in response to an injury there is an adaptive process and the cell recovers. Interestingly a by-product of UPR is activation of nuclear factor- $\mathrm{k}$, which is important in inflammation. However, if the adaptive response fails then a default pathway of cell death is activated (fig. 6b). CHOP is an enzyme that is critically involved in this process as it is implicated in both cellular arrest and apoptosis. Prolonged UPR also leads to activation of caspases that are responsible for UPR-induced cell death. It is unclear which caspases are active in this process and whether they differ between cell types. MulugETA et al. [17] found that the exon 4 deletion mutant, an SP-C BRICHOS domain mutant protein that causes ER stress and inhibits proteasome function, also activates caspase-3-mediated apoptosis.
Histological studies on lung sections from patients with UIP show evidence of BiP expression, notably over fibroblastic foci, in a significant number of patients. There is also evidence for CHOP expression in UIP, also in the area of fibroblastic foci. In contrast, there is no evidence of BiP or $\mathrm{CHOP}$ expression in the epithelium of patients with hypersensitivity pneumonitis. While the presence of BiP is indicative of UPR in IPF patients, it does not reveal if a cell is able to adapt or not. However, when CHOP is upregulated in UIP it is clear that the ER-stress pathway is projected towards URP-induced apoptosis. Furthermore, the presence of phospho-elF $2 \alpha$ expression in UIP cases, again above the fibroblastic foci, strongly suggests that there is a halt to protein translation in such cases.

Although research into the importance of misfolded proteins and the induction of UPR-mediated apoptosis in AECs in response to abnormal protein production and aggregation in IPF is still in its infancy, there are data from independent sources to suggest that it may be important in the pathogenesis of IPF (fig. 2).

\section{CONCLUSIONS}

There is substantial evidence to show that the epithelium is altered in fibroblastic foci in UIP, and that it is a pivotal regulator of lung injury, inflammation and repair. Activation of AECs results in the release of a number of growth factors, 
a)

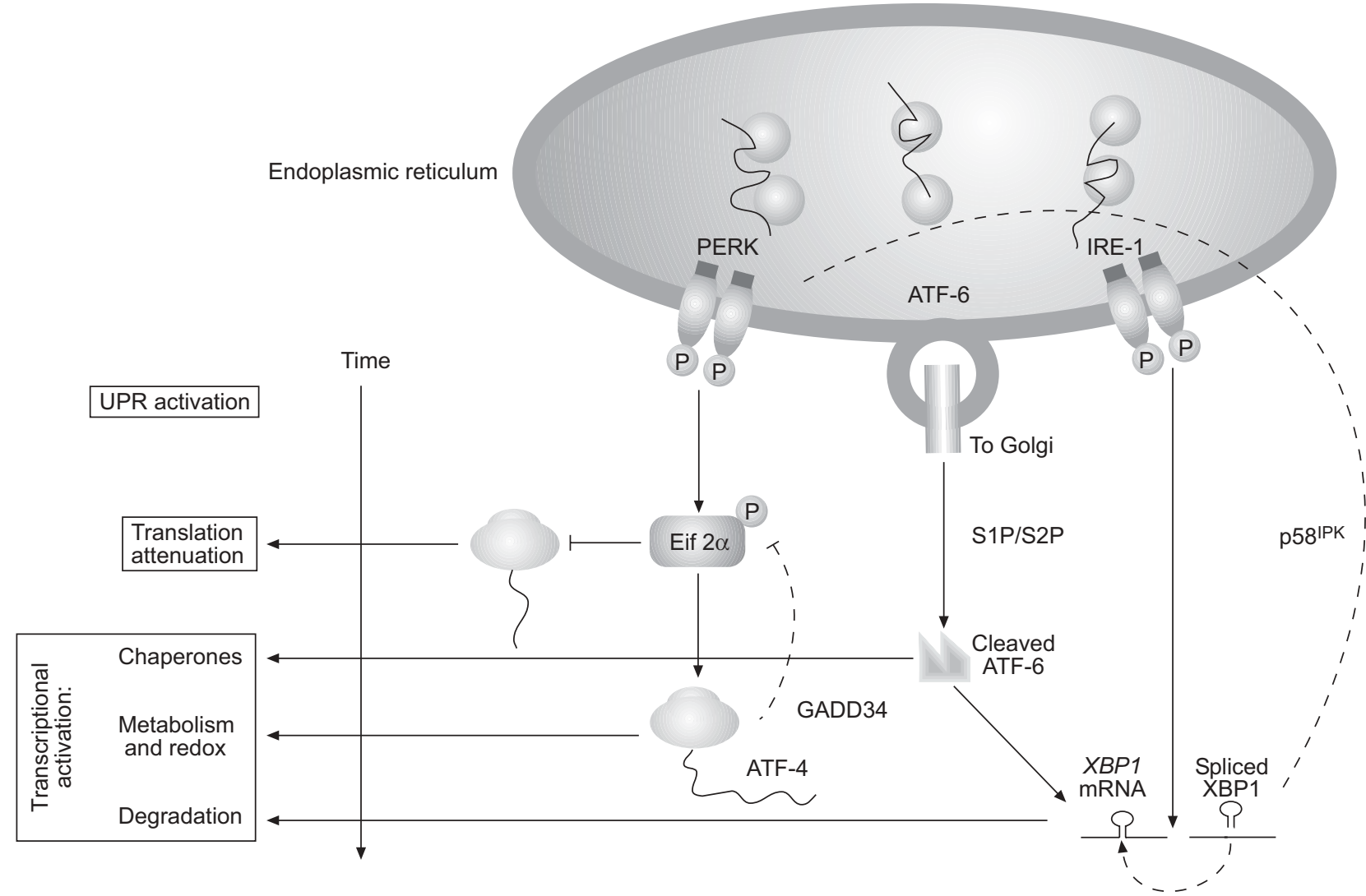

b)

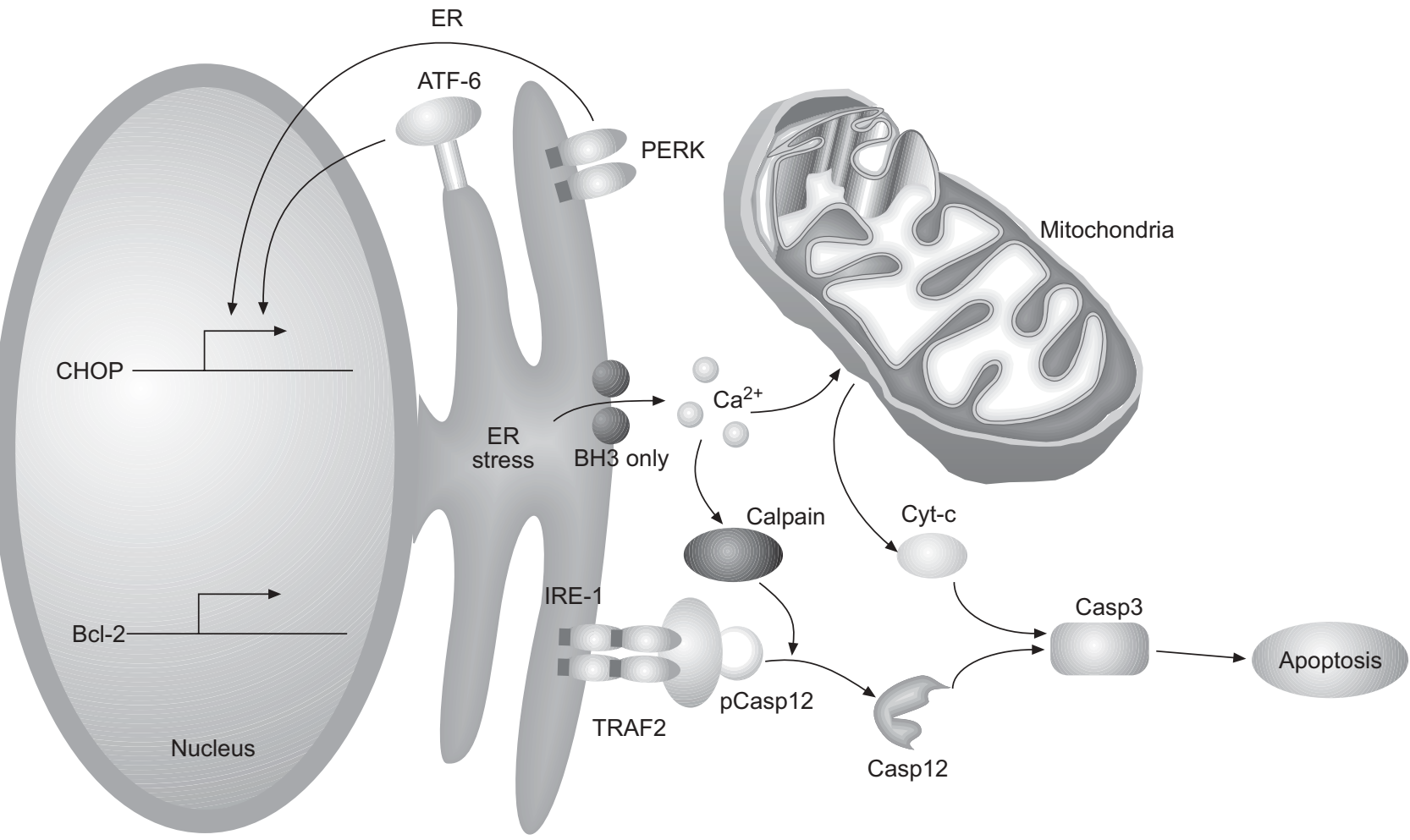

FIGURE 6. Diagram of a) the unfolded protein response (UPR) pathway and b) endoplasmic reticulum (ER) stress and UPR-induced cell death. PERK: protein kinase Rrelated ER kinase; ATF: activating transcription factor; IRE: inositol requiring kinase; P: phosphate: Eif 2 $\alpha$ : eukaryotic translation initiation factor 2 $\alpha$; S1P/S2P: site-1 protease/ site-2 protease; GADD34: growth arrest and DNA damage inducible gene 34; CHOP: CCAAT/enhancer binding protein homologous protein; BH3-only: Bcl-2 homology; TRAF2: tumour necrosis factor receptor-associated factor 2; Casp: caspase apoptosis-related cystein protease; Cyt-c: cytochrome c. Reproduced from [18] with permission from the publisher. 
coagulants, chemotactic factors for fibroblasts, ET-1 and other products of epithelial cell injury. This in turn creates a suitable environment for fibroblast recruitment. Although the source of fibroblasts in the UIP lesion is of uncertain origin, there is evidence to suggest that EMT may account for at least some of their presence in fibroblastic foci. Fibroblasts, whether recruited locally via EMT or from distant sites, then become myofibroblasts. These intra-alveolar and interstitial myofibroblasts secrete ECM proteins, predominantly collagen. ECM deposition leads to the development of clinical symptoms and the adverse clinical sequelae associated with IPF.

Targeting epithelial-fibroblast interactions is likely to be critical in developing new therapies for idiopathic pulmonary fibrosis.

\section{ADDENDUM}

There has been additional recent information from LAWSON et al. [19] that the endoplasmic reticulum stress and unfolded protein response is activated in idiopathic pulmonary fibrosis and may be associated with herpesvirus infection.

\section{REFERENCES}

1 American Thoracic Society, European Respiratory Society, American Thoracic Society. American Thoracic Society/ European Respiratory Society International Multidisciplinary Consensus Classification of the Idiopathic Interstitial Pneumonias. Am J Respir Crit Care Med 2002; 165: 277-304.

2 Selman M, King TE, Pardo A. Idiopathic pulmonary fibrosis: prevailing and evolving hypotheses about its pathogenesis and implications for therapy. Ann Intern Med 2001; 134: 136-151.

3 Noble PW, Homer RJ. Idiopathic pulmonary fibrosis: new insights into pathogenesis. Clin Chest Med 2004; 25: 749-758.

4 Raghu G, Chang J. Idiopathic pulmonary fibrosis: current trends in management. Clin Chest Med 2004; 25: 621-636.

5 Selman M, Thannickal VJ, Pardo A, Zisman DA, Martinez FJ, Lynch JP 3rd. Idiopathic pulmonary fibrosis; pathogenesis and therapeutic approaches. Drugs 2004; 64: 405-430.

6 Noble PW, Homer RJ. Back to the future: historical perspective on the pathogenesis of idiopathic pulmonary fibrosis. Am J Respir Cell Mol Biol 2005; 33: 113-120.

7 Hagimoto N, Kuwano K, Nomoto Y, Kunitake R, Hara N. Apoptosis and expression of FAS/FAS ligand mRNA in bleomycin-induced pulmonary fibrosis in mice. Am J Respir Cell Mol Biol 1997; 16: 91-101.

8 Li X, Rayford H, Uhal BD. Essential roles for angiotensin receptor AT1a in bleomycin-induced apoptosis and lung fibrosis in mice. Am J Pathol 2003; 163: 2523-2530.

9 Wang R, Ibarra-Sunga O, Pick R, Uhal BD. Abrogation of bleomycin-induced epithelial apoptosis and lung fibrosis by captopril or by a caspase inhibitor. Am J Physiol Lung Cell Mol Physiol 2000; 279: L143-L151.

10 Budinger GRS, Mutlu GM, Eisenbart J, et al. Propapoptotic Bid is required for pulmonary fibrosis. Proc Natl Acad Sci USA 2006; 103: 4604-4509.

11 Plataki M, Koutsopoulos AV, Darivianaki K, Delides G, Siafakas NM, Bouros D. Expression of apoptotic and antiapoptotic markers in epithelial cells in idiopathic pulmonary fibrosis. Chest 2005; 127: 266-274.

12 Pittet JF, Griffiths MJD, Gaiser T, et al. TGF- $\beta$ is a critical mediator of acute lung injury. J Clin Invest 1997; 100: 768-776.

13 Willis BC, Liebler JM, Luby-Phelps K, et al. Induction of epithelial-mesenchymal transition in alveolar epithelial cells by transforming growth factor- $\beta 1$. Am J Pathol 2005; 166: 1321-1332.

14 Kim KK, Kugler MC, Wolters PJ, et al. Alveolar epithelial cell mesenchymal transition develops in vivo during pulmonary fibrosis and is regulated by the extracellular matrix. Proc Natl Acad Sci USA 2006; 103: 13180-13185.

15 Jain R, Shaul PW, Borok Z, Wills BC. Endothelin-1 induces alveolar epithelial-mesnechymal transition through endothelin type A receptor-mediated production of TGF- $\beta 1$. Am J Respir Cell Mol Biol 2007; 37: 38-47.

16 Zeitlin PL, Gail DB, Banks-Schlegel S. Protein processing and degradation in pulmonary health and disease. Am J Respir Cell Mol Biol 2003; 29: 642-645.

17 Mulugeta S, Maguire JA, Newitt JL, Russo SJ, Kotoashvili A, Beers MF. Misfolded BRICHOS SP-C mutant proteins induce apoptosis via caspase-4- and cytochrome C-related mechanisms. Am J Physiol Lung Cell Mol Physiol 2007; 293: L720-L729.

18 Rutkowski DT, Kaufman RJ. A trip to the ER: coping with stress. Trends Cell Biology 2004; 14: 20-28.

19 Lawson WE, Crossno PF, Polosukhin VV, et al. Endoplasmic reticulum stress in alveolar epithelial cells is prominent in IPF: association with altered surfactant protein processing and herpesvirus infection. Am J Physiol Lung Cell Mol Physiol 2008; 294: L1119-L1126. 